\title{
КОМПЛЕКСНЫЙ АНАЛИЗ
} ПОЛИМОРФИЗМА В ПРОМОТОРНЫХ УЧАСТКАХ ГЕНОВ ЦИТОКИНОВ IL-1B T-31C, IL-6 G-174C, TNFA G-238A, TNFA G-308A, TNFA C-863A, IL-4 C-590T И IL-10 C-592 $\alpha$ В ПРОГНОЗЕ ЭФФЕКТА ОТ ЛЕЧЕНИЯ РЕВМАТОИДНОГО АРТРИТА

\section{Коненков В.И., Прокофьев В.Ф., Шевченко А.В., Голованова О.В., Зонова Е.В., Королев М.А., Леонова Ю.Б.}

Научно-исследовательский институт клинической и экспериментальной лимфологии Сибирского отделения РАМН, лаборатория клинической иммуногенетики, г. Новосибирск

Резюме. В статье приводятся результаты комплексного анализа частот встречаемости большого числа сочетаний генотипов в трех-, четырех-, пяти- и шестилокусных комбинациях аллельных вариантов генов цитокинов IL-1B T-31C, IL-6 G-174C, TNFA G-238A, TNFA G-308A, TNFA C-863A, IL-4 C-590T и IL-10 C-592A среди 104 пациентов с ревматоидным артритом, получавших базисную терапию с применением метотрексата. Показана зависимость эффекта терапии, определяемого по снижению индекса DAS28 к 24 неделям от начала лечения, от исследованных генетических комбинаций в геноме пациента. Выявлена связь интенсивности развития лекарственных осложнений с «цитокиновым генотипом» пациента. Проводится анализ полученных результатов в связи с имеющимися данными об ассоциированности уровня продукции провоспалительных и антивоспалительных цитокинов с полиморфизмом промоторных участков их генов. Предполагается, что при дальнейших клинических исследованиях полученные результаты могут быть использованы в качестве фармакогенетических критериев выбора максимально эффективной индивидуализированной терапии с учетом генотипа пациента с ревматоидным артритом.

Ключевые слова: ревматоидный артрит, полиморфизм генов цитокинов, прогноз эффекта лечения РА.

Konenkov V.I., Prokof'ev V.F., Shevchenko A.V., Golovanova O.V., Zonova E.V., Korolev M.A., Leonova J.B.

COMBINATION ANALYSIS OF CYTOKINE GENE PROMOTER POLYMORPHISMS OF IL1B T-31C, IL-6 G-174C, TNFA G-238A, TNFA G-308A, TNFA C-863A, IL4 C-590T, IL-10 C-592A FOR PREDICTION

Адрес для переписки:

Прокофьев Виктор Федорович

НИИ клинической и экспериментальной лимфологии СО РАМН

3630117, г. Новосибирск, ул. Академика Тимакова, 2.

Тел.: (383) 227-01-94.

Факс: (383) 227-01-96.

E-mail:vprok@ngs.ru
OF TREATMENT EFFICIENCY IN RHEUMATOID ARTHRITIS

Abstract. The article contains results concerning combination frequency analysis for a wide number of three- to six-locus constellations of cytokine gene alleles, i.e., IL-1B T-31C, IL-6 G-174C, TNFA G-238A, TNFA G-308A, TNFA C-863A, IL-4 C-590T, IL-10 C-592A. The study was performed 
in a group of 104 patients with rheumatoid arthritis (RA) who were subject to basic therapy with methotrexate. An association was found between certain individual multi-locus allele combinations, and efficiency of the therapy, as defined by decrease in DAS28 index following 24 week of methotrexate treatment. An association was demonstrated between adverse effects of drugs used, and a "cytokine genotype" of the patients. Analysis of the results was carried out with respect to correlations between the levels of pro- and anti-inflammatory cytokine production and promoter polymorphisms of respective genes. We assume that further clinical research will allow to use our results as pharmacogenetic criteria in order to choose more effective personalized therapies, taking into account the genotypes of RA patients. (Med. Immunol., vol. 12, N 4-5, pp 361-374)

Keywords: rheumatoid arthritis, treatment efficiency, cytokines, gene polymorphism, prediction.

\section{Введение}

Патогенез ревматоидного артрита (РА), несмотря на многолетние и многоцентровые исследования, до настоящего времени не совсем понятен. Большим достижением медицинской иммунологии в области ревматологии, несомненно, является расшифровка механизмов развития воспалительного процесса в суставах, связанного с локальными и системными эффектами цитокинов. Это открытие привело к созданию нового класса лекарственных препаратов биологических агентов, или генно-инженерных препаратов, в основе которых лежат моноклональные антитела к различным эпитопам цитокинов или их рецепторам.

Воспаление, лежащее в основе патологического процесса при РА, во многом зависит от баланса продукции цитокинов с провоспалительной и антивоспалительной активностью $[8$, 21]. Казалось бы, очевидная мысль об использовании показателей их содержания в сыворотке крови не нашла своего развития на практике. Основанием этому послужило наличие чрезвычайно широких колебаний концентраций цитокинов в сыворотке крови, как здоровых лиц, так и пациентов с РА и зависимость их концентраций не столько от активности ревматоидного воспаления, сколько от множества динамичных факторов. Среди последних необходимо отметить циркуляцию в кровеносном русле растворимых цитокиновых рецепторов, уровень экспрессии последних на клеточных мембранах, уровень продукции антагонистов и агонистов цитокинов, активность сывороточных рестриктаз, наличие сплайсированных форм цитокинов и многое другое.

Наличие в регуляторных участках генов цитокинов полиморфных позиций, однонуклеотидная замена в которых приводит к различным уровням продукции клеткой биологически активных молекул соответствующих цитокинов, позволяет использовать генотипирование для определения базового уровня продукции цитокинов у индивида, не прибегая к исследованию постоянно меняющейся их концентрации в сыворотке крови [22, 33, 36].

Зависимость уровня продукции клетками цитокинов от точечных замен в промоторных участках генов цитокинов, делает более понятным ассоциированность аллельных вариантов этих генов с РА и ставит их в первый ряд кандидатных генов для фармагенетических исследований в ревматологии $[10,17,23]$.

Такие попытки научных центрах различных стран неоднократно предпринимаются, однако их эффективность требует проверки клинической практикой [15, 28, 34, 37]. В России таких исследований явно недостаточно, тем более что этногенетические особенности населения такой многонациональной страны, как Российская федерация, требуют критичного отношения к использованию подобных данных, полученных на других европеоидных, монголоидных и негроидных популяциях [6].

По современным стандартам целью фармакотерапии РА является достижение полной (или хотя бы частичной) ремиссии. Следует отметить, что терапия должна начинаться как можно раньше, желательно в течение 3-6 месяцев от начала болезни [16].

В настоящее время метотрексат (МТ) получил статус «золотого стандарта» среди всех БПВП, использующихся для лечения РА [31]. Так же широко используются другие препараты - лефлуномид, сульфасалазин, гидроксихлорохин и различные комбинированные схемы $[4,27]$.

Принципиально важным является оптимизация выбора лекарственного средства с предварительной оценкой возможной чувствительности к выбранному методу лечения. В настоящее время подбор препарата осуществляется эмпирически, а мультифакториальность природы ревматоидного артрита обуславливает и различную чувствительность пациентов к тера- 
пии. Точечный выбор эффективного препарата или метода лечения в короткие сроки улучшает непосредственный (подавление симптомов) и даже отдаленный (снижение риска инвалидности) прогноз у многих пациентов.

Нами исследован полиморфизм генов цитокинов с провоспалительной активностью IL-1B T-31C, IL-6 G-174C, TNFA G-238A, TNFA G-308A, TNFA C-863A, а также генов цитокинов с противовоспалительной активностью IL-4 C-590Т и IL-10 C-592А в их промоторных участках, ассоциированных с высоким или низким уровнем их продукции у пациентов с различной эффективностью базисной терапии.

\section{Материалы и методы}

Пациенты. В исследование включено 104 пациента с РА (93 женщины и 11 мужчин, средний возраст 53,38 $\pm 13,55$ лет). Диагноз РА соответствовал критериям Американской ревматологической ассоциации (1987). Продолжительность заболевания составила 9,18 $\pm 8,97$ лет. Распределение больных по рентгенологическим стадиям [35] было следующим: II стадия - 36,54 \%, III $36,54 \%$ и IV - 26,92\% . Преобладали пациенты со 2 и 3 классом функциональной недостаточности суставов (ACR, 1992 г.), наличием экстраартикулярных проявлений болезни $(79,81 \%)$. Степень активности с учетом индекса DAS28 (Desease Activity Score, оценка 28 суставов) определена как умеренная в $34,62 \%$ случаев, в $65,38 \%$ - как высокая и в среднем составила $5,53 \pm 1,32$. Уровень HAQ - 1,97 $\pm 1,12$. Позитивными по ревматоидному фактору (РФ) определены $87,65 \%$ обследованных, по антителам к циклическому цитруллинированному пептиду (а-ЦЦП) - 83,5\%.

Ранее проводимая терапия была неэффективна или, в связи с коротким анамнезом болезни и верифицированием диагноза РА, иммуносупрессивная терапия назначалась впервые. Использовался метотрексат в дозе 10-17,5 мг в неделю $(69,23 \%)$ или сульфасалазин 2 г в сутки $(30,77 \%)$. Эффективность терапии через 24 недели от ее начала оценивалась по критериям EULAR с учетом разницы показателя DAS28, оцененного в динамике. Эффект лечения оценивался как высокий при значениях DAS28 $<3,2$ в тех случаях, когда он снижался в процессе лечения более, чем на 1,2 балла. Если динамика DAS28 была в пределах 0,6 до 1,2 баллов, эффект считался удовлетворительным, при разнице значений DAS28 $<0,6$ эффекта не было. При значе- ниях DAS28 от 3,2 до 5,1 баллов (при сохраненной умеренной активности заболевания) эффект терапии оценивался как удовлетворительный, если показатели DAS28 уменьшались не менее чем на 0,6 баллов, у остальных пациентов этой группы эффект терапии не считался значимым. Если активность заболевания по DAS28 после лечения оставалась высокой (более 5,1 балла), то удовлетворительным эффектом терапии можно было считать только значительную его динамику, т.е. уменьшение DAS28 более чем на 1,2 балла [20].

Методы исследования полиморфизма генов. Геномную ДНК выделяли из ядросодержащих клеток 500 мкл переферической крови при помощи набора реагентов производства ООО «Лаборатория Медиген» (г. Новосибирск). Исследовались шесть полиморфизмов, локализованных в промоторных регионах генов интерлейкинов: TNFA в позициях C-863A, G-308A, G-238A, IL-1B T-31C, IL-4 C-590T, IL-6 G-174C, IL-10 C-592A. Анализ исследуемых полиморфизмов проводился с использованием метода рестрикционного анализа продуктов амплификации (ПДРФ-анализ). Амплификация специфических участков генома проводилась с использованием праймеров и параметров температурных циклов, описанных в литературе: TNFA [13, 24 , 30], IL-1B [38], IL-4 [14], IL-6 [18], IL-10 [26]. Продукты амплификации подвергались рестрикции соответствующими эндонуклеазами: в случае генотипирования полиморфизма TNFA C-863A - Bst BAI, TNFA G-308A - Bsp19 I , TNFA G-238A - Msp I, IL-1B T-31C - Alu I, IL-4 C-590T - Bme 18 I, IL-6 G-174C - SfaN I, IL10 C-592A - RsaI («СибЭнзим», Новосибирск). Гидролиз продуктов амплификации проводили в течение 12 часов при оптимальной для фермента температуре. Продукты рестрикции разделяли с помощью электрофореза в $2 \%$ агарозном геле. В качестве маркера длин фрагментов ДНК использовали плазмиду pUC19, расщепленную рестриктазой MspI («СибЭнзим», Новосибирск).

Статистическая обработка данных. При статистическом анализе результатов исследований использовали такие показатели как частота встречаемости генов, генотипов и их комбинаций, отношение шансов (OR), доверительный интервал (CI) для OR и диагностический вес признаков (DK).

Частоту аллелей генов цитокинов вычисляли методом прямого подсчета по формуле: 
$\mathrm{f}=\mathrm{n} / 2 \mathrm{~N}$, где $\mathrm{n}-$ количество раз встречаемости аллеля (у гомозигот он учитывался дважды), $2 \mathrm{~N}$ - удвоенная численность обследованных.

Распределение генотипов по исследованным полиморфным локусам проверяли на соответствие равновесию Харди-Вайнберга согласно рекомендациям Вейр Б. [1].

Частоту встречаемости отдельных генотипов и их комбинаций определяли как процентное отношение индивидов, несущих генотип (комбинацию генотипов), к общему числу обследованных в группе по формуле: $\mathrm{f}=\mathrm{n} / \mathrm{N}$, где $\mathrm{n}$ - количество раз встречаемости генотипа (комбинации генотипов), N - численность обследованных.

Статистическую оценку силы ассоциации генов, генотипов и их комбинаций с клиническими эффектами терапии проводили по показате- лю OR (odds ratio - отношение шансов события в одной группе к шансам этого же события в другой группе) с расчетом 95\% доверительного интервала (95\% Confidence Interval - 95\% CI) $[9,25]$.

Достоверность (P) ассоциаций и различий частот распределения изучаемых признаков в альтернативных группах определяли по критерию $\chi^{2}$ с поправкой Йетса на непрерывность и двустороннему варианту точного метода Фишера для четырехпольных таблиц $[3,11]$.

Отбор генетических комплексов, пригодных для индивидуального прогнозирования эффективности терапии, проводился на основе значений трех статистических критериев: двусторонний вариант точного метода Фишера; информационная мера Кульбака (Jku); диагностический (прогностический) коэффициент

\section{ТАБЛИЦА 1. ЧАСТОТЫ КОМБИНАЦИЙ АЛЛЕЛЕЙ ГЕНОВ ЦИТОКИНОВ У ПАЦИЕНТОВ РА С ВЫСОКОЙ} ЭФФЕКТИВНОСТЬЮ ТЕРАПИИ

\begin{tabular}{|c|c|c|c|c|c|}
\hline \multirow[b]{2}{*}{ Комбинации аллелей } & \multicolumn{2}{|c|}{$\begin{array}{c}\text { Частота среди больных } \\
\text { РА (в \%) } \\
\end{array}$} & \multirow{2}{*}{$\begin{array}{c}\text { Прогности- } \\
\text { ческий ко- } \\
\text { эфффициент }\end{array}$} & \multirow{2}{*}{$\begin{array}{l}\text { Значимость } \\
\text { различий (p) }\end{array}$} & \multirow{2}{*}{$\begin{array}{l}\text { Специфич- } \\
\text { ность }\end{array}$} \\
\hline & $\begin{array}{c}\text { высокая } \\
\text { эффектив- } \\
\text { ность }\end{array}$ & $\begin{array}{l}\text { отсутствие } \\
\text { эфрфекта }\end{array}$ & & & \\
\hline IL-4590CT/IL6174GG/IL-1B31CC & 12,90 & 0,00 & 9,8 & 0,0495 & 100,00 \\
\hline IL-4590CC/TNFA308GA/TNFA863CC & 20,00 & 2,94 & 8,3 & 0,0444 & 97,06 \\
\hline IL6174GC/TNFA308GA/TNFA863CC & 20,00 & 3,03 & 8,2 & 0,0391 & 96,97 \\
\hline IL-6174GG/TNFA863CC/IL-1B31CC & 13,33 & 0,00 & 9,9 & 0,0460 & 100,00 \\
\hline IL-10592CC/TNFA308GA/TNFA863CC & 20,00 & 2,94 & 8,3 & 0,0444 & 97,06 \\
\hline $\begin{array}{l}\text { IL-4590CT/IL6174GG/TNFA238GG/ } \\
\text { IL-1B31CC }\end{array}$ & 12,90 & 0,00 & 9,8 & 0,0495 & 100,00 \\
\hline $\begin{array}{l}\text { IL-4590CT/IL6174GG/TNFA308GG/ } \\
\text { IL-1B31CC }\end{array}$ & 12,90 & 0,00 & 9,8 & 0,0495 & 100,00 \\
\hline $\begin{array}{l}\text { IL-4590CT/IL6174GG/TNFA863CC/ } \\
\text { IL-1B31CC }\end{array}$ & 13,33 & 0,00 & 9,9 & 0,0460 & 100,00 \\
\hline $\begin{array}{l}\text { IL-6174GC/TNFA238GG/TNFA308GA/ } \\
\text { TNFA863CC }\end{array}$ & 20,00 & 3,03 & 8,2 & 0,0391 & 96,97 \\
\hline $\begin{array}{l}\text { IL-6174GG/TNFA238GG/TNA863CC/ } \\
\text { IL-1B31CC }\end{array}$ & 13,33 & 0,00 & 9,9 & 0,0460 & 100,00 \\
\hline $\begin{array}{l}\text { IL-6174GG/TNFA308GG/TNFA863CC/ } \\
\text { IL-1B31CC }\end{array}$ & 13,33 & 0,00 & 9,9 & 0,0460 & 100,00 \\
\hline $\begin{array}{l}\text { IL-6174GG/TNFA308GG/TNFA863CC/ } \\
\text { IL-1B31CC }\end{array}$ & 13,33 & 0,00 & 9,9 & 0,0460 & 100,00 \\
\hline $\begin{array}{l}\text { IL-4590CT/IL6174GG/TNFA238GG/ } \\
\text { TNFA308GG/IL-1B31CC }\end{array}$ & 12,90 & 0,00 & 9,8 & 0,0495 & 100,00 \\
\hline $\begin{array}{l}\text { IL-4590CT/IL6174GG/TNFA238GG/ } \\
\text { TNFA863CC/IL-1B31CC }\end{array}$ & 13,33 & 0,00 & 9,9 & 0,0460 & 100,00 \\
\hline $\begin{array}{l}\text { IL-4590CT/IL6174GG/TNFA308GG/ } \\
\text { TNFA863CC/IL-1B31CC }\end{array}$ & 13,33 & 0,00 & 9,9 & 0,0460 & 100,00 \\
\hline $\begin{array}{l}\text { IL-6174GG/TNFA238GG/TNFA308GG/ } \\
\text { TNFA863CC/IL-1B31CC }\end{array}$ & 13,33 & 0,00 & 9,9 & 0,0460 & 100,00 \\
\hline
\end{tabular}


по формуле: $\mathrm{DK}=10 \lg \left(\mathrm{P}_{1} / \mathrm{P}_{2}\right)$, где $\mathrm{P}_{1}$ и $\mathrm{P}_{2}$ - частота прогностического признака в сравниваемых группах [3, 5, 7]. Необходимо напомнить, что согласно данному методу анализа, при достижении прогностического коэффициента величины 12,8 вероятность реализации прогноза составляет $95 \%$.

В качестве операционных характеристик генетических комплексов в качестве прогностических критериев использовали также показатели чувствительности, специфичности, безошибочности, предсказательной ценности положительного результата и предсказательной ценности отрицательного результата по стандартным формулам $[2,7,9,12]$.

\section{Результаты}

По результатам проведенного 24 недельного лечения пациенты были разделены на группы с высокой эффективностью проведенного лечения, с отсутствием клинически значимого эффекта от проведенной терапии и с развитием неблагоприятных побочных реакций.

С высоким ответом на болезньмодифицируюшую терапию зафиксировано 29,81\% пациентов, с отсутствием эффекта - 32,69\%, у остальных $(37,5 \%)$ - умеренная эффективность используемых препаратов. У 64 пациентов $(61,54 \%)$ в период 24 недельного наблюдения отмечена хорошая переносимость болезньмодифицирующей терапии, у 21 пациента $(20,19 \%)$ зафиксированы незначительные проявления непереносимости, которые не послужили причиной отмены иммуносупрессивной терапии. У 19 больных $(18,27 \%)$ развились гепатотоксические реакции, лейкопения или тромбоцитопения, потребовавшие отмены используемого метода лечения.

У части пациентов с РА, в генотипе которых присутствуют определенные комбинации аллельных вариантов промоторных участков генов цитокинов, с различной частотой реализуется высокая эффективность базисной терапии (табл. 1). При анализе результатов в данных выборках пациентов нами проанализированы частоты встречаемости 16362 возможных сочетаний генотипов 2-7 локусных комбинаций, из которых достоверно различающимися $(\mathrm{p}<0,05)$ при использовании одностороннего варианта точного метода Фишера оказалось 21, а при использовании двустороннего варианта -18 комбинаций генотипов. Этим пациентам с наличием в геноме, представленных в таблице 1 , генетических комбинаций можно рекомендо- вать продолжение курсовой болезньмодифицирующей терапии, без необходимости перехода к дорогостоящей терапии генно-инженерными препаратами.

Как правило, среди таких генетических комбинаций присутствует генотип IL-6 -174GG, acсоциированный с высоким уровнем продукции этого провоспалительного цитокина, обладающего выраженными системными и суставными эффектами, во многом определяющими клиническую картину течения РА [29].

С другой стороны, среди этих пациентов достаточно часто выявляется генотип IL-1B -31CC, ассоциированный с низкой продукцией IL- $1 \beta$, характеризующегося провоспалительной активностью. Этот цитокин, продуцируемый в основном макрофагами, активно инициирует начальные фазы развития системного воспалительного процесса и, по мере развития заболевания, главенствующая роль в его поддержании переходит к IL-6.

Среди больных РА в этой группе также достаточно часто выявляются гомозиготные генотипы TNFA в позициях $-863,-308$ и -238 , аллели $\mathrm{G}$ и $\mathrm{C}$ в которых также ассоциированы с низкой продукцией еще одного цитокина с выраженной провоспалительной активностью - фактора некроза опухолей $\alpha$.

Результаты анализа полиморфизма в промоторных зонах генов цитокинов с противовоспалительной активностью не дают четких результатов. У пациентов данной группы, в основном, выявляются гетерозиготные генотипы IL-4 -590 CT, ассоциированные как с низкой, так и с высокой способностью клеток продуцировать этот цитокин. В одной комбинации выявлен гомозиготный вариант гена IL-10 -592СС, ассоциированный с высокой продукцией данного цитокина, способного подавлять течение воспалительного процесса.

Возможность прогнозировать высокую эффективность наиболее часто применяемой базисной терапии, несомненно, важна для любого практикующего ревматолога, однако не менее значимо предвидеть возможность ее неэффективности. Хорошо известно, что потеря временного «окна терапевтических возможностей» чрезвычайно важна для успеха в определении терапевтической стратегии длительного лечения РА. Для исследования этого момента, нами проведен анализ распределения различных комбинаций аллелей генов цитокинов в группе пациентов с РА, которым 24 недельная терапия 
ТАБЛИЦА 2. ЧАСТОТЫ КОМБИНАЦИЙ АЛЛЕЛЕЙ ГЕНОВ ЦИТОКИНОВ У ПАЦИЕНТОВ РА С ОТСУТСТВИЕМ ЭФФЕКТА ТЕРАПИИ

\begin{tabular}{|c|c|c|c|c|c|}
\hline \multirow[b]{2}{*}{ Комбинации аллелей } & \multicolumn{2}{|c|}{$\begin{array}{c}\text { Частота среди боль- } \\
\text { ных РА (в \%) }\end{array}$} & \multirow{2}{*}{$\begin{array}{c}\text { Прогностиче- } \\
\text { ский коэфффи- } \\
\text { циент }\end{array}$} & \multirow{2}{*}{$\begin{array}{c}\text { Значи- } \\
\text { мость раз- } \\
\text { личий (р) }\end{array}$} & \multirow{2}{*}{$\begin{array}{c}\text { Специфичч- } \\
\text { ность }\end{array}$} \\
\hline & $\begin{array}{l}\text { отсут- } \\
\text { ствие эфр- } \\
\text { фекта }\end{array}$ & $\begin{array}{l}\text { наличие } \\
\text { эфффекта }\end{array}$ & & & \\
\hline TNFA308GA/TNFA863CA & 11,76 & 1,47 & 9,0 & 0,0412 & 98,53 \\
\hline IL-4590CC/TNFA308GA/TNFA863CA & 11,76 & 0,00 & 12,5 & 0,0109 & 100,00 \\
\hline IL-6174GC/IL-10592CC/IL-1B31TT & 27,27 & 10,00 & 4,4 & 0,0389 & 90,00 \\
\hline IL-6174CC/IL-10592CA/IL-1B31TC & 9,09 & 0,00 & 11,7 & 0,0309 & 100,00 \\
\hline IL-6174GC/TNFA238GG/TNFA308GG & 48,48 & 27,14 & 2,5 & 0,0351 & 72,86 \\
\hline IL-6174GC/TNFA308GG/TNFA863CC & 45,45 & 19,12 & 3,8 & 0,0062 & 80,88 \\
\hline IL-6174GC/TNFA308GG/IL-1B31TT & 36,36 & 14,29 & 4,1 & 0,0123 & 85,71 \\
\hline IL-6174CC/TNFA863CA/IL-1B31TC & 12,12 & 1,47 & 9,2 & 0,0381 & 98,53 \\
\hline IL-10592CA/TNFA863CA/IL-1B31TC & 8,82 & 0,00 & 11,4 & 0,0349 & 100,00 \\
\hline $\begin{array}{l}\text { IL-4590CC/IL6174CC/TNFA863CA/IL- } \\
\text { 1B31TC }\end{array}$ & 9,09 & 0,00 & 11,6 & 0,0327 & 100,00 \\
\hline $\begin{array}{l}\text { IL-4590CC/TNFA238GG/TNFA308GA/ } \\
\text { TNFA863CA }\end{array}$ & 8,82 & 0,00 & 11,4 & 0,0349 & 100,00 \\
\hline $\begin{array}{l}\text { IL-4590CC/TNFA308GA/TNFA863CA/ } \\
\text { IL1B31TT }\end{array}$ & 8,82 & 0,00 & 11,4 & 0,0349 & 100,00 \\
\hline $\begin{array}{l}\text { IL-6174GC/IL-10592CC/TNFA238GG/ } \\
\text { TNFA308GG }\end{array}$ & 30,30 & 11,43 & 4,2 & 0,0263 & 88,57 \\
\hline $\begin{array}{l}\text { IL-6174GC/IL-10592CC/TNA238GG/ } \\
\text { IL-1B31TT }\end{array}$ & 27,27 & 8,57 & 5,0 & 0,0174 & 91,43 \\
\hline $\begin{array}{l}\text { IL-6174CC/IL-10592CA/TNAF238GG/ } \\
\text { IL-1B31TC }\end{array}$ & 9,09 & 0,00 & 11,7 & 0,0309 & 100,00 \\
\hline $\begin{array}{l}\text { IL-6174GC/IL-10592CC/TNFA308GG/ } \\
\text { TNFA863CC }\end{array}$ & 27,27 & 8,82 & 4,9 & 0,0332 & 91,18 \\
\hline $\begin{array}{l}\text { IL-6174GC/IL-10592CC/TNFA308GG/ } \\
\text { IL-1B31TT }\end{array}$ & 24,24 & 5,71 & 6,3 & 0,0168 & 94,29 \\
\hline $\begin{array}{l}\text { IL-6174CC/IL10592CA/TNFA863CA/ } \\
\text { IL-1B31TC }\end{array}$ & 9,09 & 0,00 & 11,6 & 0,0327 & 100,00 \\
\hline $\begin{array}{l}\text { IL-6174GC/TNFA238GG/TNFA308GG/ } \\
\text { TNAF863CC }\end{array}$ & 45,45 & 17,65 & 4,1 & 0,0043 & 82,35 \\
\hline $\begin{array}{l}\text { IL-6174GC/TNFA238GG/TNFA308GG/ } \\
\text { IL-1B31TT }\end{array}$ & 36,36 & 12,86 & 4,5 & 0,0087 & 87,14 \\
\hline $\begin{array}{l}\text { IL-6174CC/TNFA238GG/TNFA863CA/ } \\
\text { IL-1B31TC }\end{array}$ & 12,12 & 1,47 & 9,2 & 0,0381 & 98,53 \\
\hline $\begin{array}{l}\text { IL-6174GC/TNFA308GG/TNFA863CC/ } \\
\text { IL-1B31TT }\end{array}$ & 33,33 & 11,76 & 4,5 & 0,0141 & 88,24 \\
\hline $\begin{array}{l}\text { IL-10592CC/TNFA238GG/TNFA308GG/ } \\
\text { TNFA863CC }\end{array}$ & 38,24 & 17,65 & 3,4 & 0,0292 & 82,35 \\
\hline $\begin{array}{l}\text { IL-10592CA/TNFA238GG/TNFA863CA/ } \\
\text { IL-1B31TC }\end{array}$ & 8,82 & 0,00 & 11,4 & 0,0349 & 100,00 \\
\hline $\begin{array}{l}\text { IL-10592CC/TNFA308GG/TNFA863CC/ } \\
\text { IL1B31TT }\end{array}$ & 23,53 & 7,35 & 5,1 & 0,0289 & 92,65 \\
\hline $\begin{array}{l}\text { IL-6174GC/IL10592CC/TNFA238GG/ } \\
\text { TNFA863CC/IL-1B31TT }\end{array}$ & 9,09 & 0,00 & 11,6 & 0,0327 & 100,00 \\
\hline $\begin{array}{l}\text { IL-6174GC/IL-10592CC/TNFA238GG/ } \\
\text { TNAF308GG/TNA863CC }\end{array}$ & 27,27 & 7,35 & 5,7 & 0,0119 & 92,65 \\
\hline
\end{tabular}




\begin{tabular}{|c|c|c|c|c|c|}
\hline \multirow[b]{2}{*}{ Комбинации аллелей } & \multicolumn{2}{|c|}{$\begin{array}{c}\text { Частота среди боль- } \\
\text { ных РА (в \%) } \\
\end{array}$} & \multirow{2}{*}{$\begin{array}{l}\text { Прогностиче- } \\
\text { ский коэфффи- } \\
\text { циент }\end{array}$} & \multirow{2}{*}{$\begin{array}{l}\text { 3начи- } \\
\text { мость раз- } \\
\text { личий (p) }\end{array}$} & \multirow[b]{2}{*}{$\begin{array}{l}\text { Специфиич- } \\
\text { ность }\end{array}$} \\
\hline & $\begin{array}{c}\text { отсут- } \\
\text { ствие эф- } \\
\text { фекта } \\
\end{array}$ & $\begin{array}{l}\text { наличие } \\
\text { эффректа }\end{array}$ & & & \\
\hline $\begin{array}{l}\text { IL-6174GC/IL10592CC/TNFA238GG/ } \\
\text { TNFA308GG/LL-1B31TT }\end{array}$ & 24,24 & 4,29 & 7,5 & 0,0043 & 95,71 \\
\hline $\begin{array}{l}\text { IL-6174GC/IL10592CC/TNFA238GG/ } \\
\text { TNFA863CC/IL-1B31TT }\end{array}$ & 21,21 & 5,88 & 5,6 & 0,0365 & 94,12 \\
\hline $\begin{array}{l}\text { IL-4590CC/IL6174CC/TNFA238GG/ } \\
\text { TNFA863CA/IL-1B31TC }\end{array}$ & 9,09 & 0,00 & 11,6 & 0,0327 & 100,00 \\
\hline $\begin{array}{l}\text { IL-6174GC/IL10592CC/TNFA308GG/ } \\
\text { TNFA863CC/IL-1B31TT }\end{array}$ & 21,21 & 2,94 & 8,6 & 0,0051 & 97,06 \\
\hline $\begin{array}{l}\text { IL-6174GC/TNFA238GG/TNFA308GG/ } \\
\text { TNFA863CC/IL-1B31TT }\end{array}$ & 33,33 & 10,29 & 5,1 & 0,0106 & 89,71 \\
\hline $\begin{array}{l}\text { IL-10592CC/TNFA238GG/TNFA308GG/ } \\
\text { TNFA863CC/LL-1B31TT }\end{array}$ & 23,53 & 5,88 & 6,0 & 0,0185 & 94,12 \\
\hline $\begin{array}{l}\text { IL-6174GC/IL-10592CC/TNFA238GG/ } \\
\text { TNFA308GG/TNFA863CC/IL1B31TT }\end{array}$ & 21,21 & 1,47 & 11,6 & 0,0015 & 98,53 \\
\hline $\begin{array}{l}\text { IL-4590CC/IL10592CC/TNFA238GG/ } \\
\text { TNFA308GG/TNFA863CC/IL1B31TT }\end{array}$ & 8,82 & 0,00 & 11,4 & 0,0349 & 100,00 \\
\hline
\end{tabular}

не принесла объективного снижения активности ревматоидного процесса, учитываемого по снижению показателя DAS28.

Проведенное исследование показало, что у части пациентов РА с наличием в геноме определенных комбинаций аллельных вариантов генов промоторных участков генов цитокинов базисная болезньмодифицирующая терапия ни в одном случае не оказывает положительного эффекта (табл. 2). При анализе результатов в данных выборках пациентов нами проанализированы частоты встречаемости 16362 возможных сочетаний генотипов 2-7 локусных комбинаций, из которых достоверно различающимися $(\mathrm{p}<0,05)$ при использовании одностороннего варианта точного метода Фишера оказалось 40, а при использовании двустороннего варианта 35 комбинаций генотипов.

Соответственно при выявлении у пациента этих генетических комбинаций встает вопрос о целесообразности проведения данного метода лечения, о необходимости перехода на терапию генно-инженерными биологическими препаратами или на интенсификацию лечения базисными противовоспалительными средствами.

Анализ частоты встречаемости повторяющихся аллелей в этой группе пациентов с РА показал, что в позиции G-174C промоторного региона гена IL-6 наиболее часто выявляется аллель C, ассоциированный с низким уровнем продукции этого основного провоспалительного цитокина.
Дальнейшее исследование полиморфизма генов провоспалительных цитокинов среди «неответчиков» на терапию показало, что из аллелей гена фактора некроза опухолей наиболее часто выявляются аллели в полиморфных позициях G-238A, G-308A, C-863A, ассоциированные с низким уровнем его продукции.

В данной группе пациентов с отсутствием эффекта от лечения наиболее часто выявляется аллель Т гена IL-1B в позиции T-31C, напротив ассоциированный с высоким уровнем продукции этого цитокина с выраженной провоспалительной активностью.

Среди проанализированных генов цитокинов с антивоспалительной активностью в полиморфных позициях их промоторных участков: IL-4 C-590T и IL-10 C-592A в основном выделены аллельные варианты, ассоциированные с низким уровнем их продукции.

Таким образом, пациенты с РА, являющиеся хорошими «ответчиками» и «неответчиками» на DMARDs терапию кардинальным образом различаются не только по комбинациям аллелей генов цитокинов с провоспалительной и антивоспалительной активностью, но и различаются по преобладанию аллелей этих генов, ассоциированных с различным уровнем продукции этих факторов регуляции воспаления.

Так в группе пациентов с выраженным эффектом от проведенного лечения преобладают аллели, ассоциированные с высокой продукцией интерлейкина-6 и низкой продукцией IL-1, 
ТАБЛИЦА 3. ЧАСТОТЫ КОМБИНАЦИЙ АЛЛЕЛЕЙ ГЕНОВ ЦИТОКИНОВ У ПАЦИЕНТОВ РА С ВЫРАЖЕННЫМ ПОБОЧНЫМ ДЕЙСТВИЕМ ТЕРАПИИ

\begin{tabular}{|l|c|c|c|c|c|}
\hline \multirow{2}{*}{ Комбинации аллелей } & \multicolumn{2}{|c|}{$\begin{array}{c}\text { Частота среди больных } \\
\text { РА(в \%) }\end{array}$} & $\begin{array}{c}\text { Прогности- } \\
\text { ческий ко- } \\
\text { эфроциент }\end{array}$ & $\begin{array}{c}\text { 3начимость } \\
\text { различий (р) }\end{array}$ & $\begin{array}{c}\text { Специфич- } \\
\text { ность }\end{array}$ \\
\cline { 2 - 5 } & $\begin{array}{c}\text { побочные } \\
\text { реакции }\end{array}$ & отсутствие & \\
\hline IL-4590CC/IL6174GC/IL10592CA & 26,32 & 7,94 & 5,2 & 0,0382 & 92,06 \\
\hline IL-4590CC/IL6174GC/TNFA308GA & 15,79 & 1,59 & 10,0 & 0,0371 & 98,41 \\
\hline $\begin{array}{l}\text { IL-4590CC/IL6174GC/IL10592CA/ } \\
\text { TNFA238GG }\end{array}$ & 26,32 & 7,94 & 5,2 & 0,0468 & 92,06 \\
\hline $\begin{array}{l}\text { IL-4590CC/IL6174GC/IL10592CA/ } \\
\text { IL1B31TC }\end{array}$ & 15,79 & 0,00 & 13,6 & 0,0109 & 100,00 \\
\hline $\begin{array}{l}\text { IL-4590CC/IL6174GC/ } \\
\text { TNFA238GG/TNFA308GA }\end{array}$ & 15,79 & 1,59 & 10,0 & 0,0371 & 98,41 \\
\hline $\begin{array}{l}\text { IL-4590CC/IL6174GC/IL10592CA/ } \\
\text { TNFA238GG/IL1B31TC }\end{array}$ & 15,79 & 0,00 & 13,6 & 0,0109 & 100,00 \\
\hline
\end{tabular}

\section{ТАБЛИЦА 4. ЧАСТОТЫ КОМБИНАЦИЙ АЛЛЕЛЕЙ ГЕНОВ ЦИТОКИНОВ У ПАЦИЕНТОВ РА С НЕЖЕЛАТЕЛЬНЫМИ ЭФФЕКТАМИ ТЕРАПИИ}

\begin{tabular}{|c|c|c|c|c|c|}
\hline \multirow{2}{*}{ Комбинации аллелей } & \multicolumn{2}{|c|}{$\begin{array}{c}\text { Частота среди больных РА } \\
\text { (в \%) }\end{array}$} & \multirow{2}{*}{$\begin{array}{c}\text { Прогности- } \\
\text { ческий ко- } \\
\text { эфрфициент }\end{array}$} & \multirow{2}{*}{$\begin{array}{l}\text { Значимость } \\
\text { различий (p) }\end{array}$} & \multirow{2}{*}{$\begin{array}{l}\text { Специфич- } \\
\text { ность }\end{array}$} \\
\hline & $\begin{array}{l}\text { нежелатель- } \\
\text { ный эффрект }\end{array}$ & отсутствие & & & \\
\hline IL-6174GC/IL10592CA & 33,33 & 12,70 & 4,20 & 0,0478 & 87,3 \\
\hline IL-6174GG/TNFA238GG & 47,62 & 22,2 & 3,30 & 0,0483 & 77,78 \\
\hline IL-6174GG/TNFA863CA & 30,00 & 6,5 & 6,70 & 0,0116 & 93,55 \\
\hline IL-4590CT/IL-6174GG/IL10592CA & 23,81 & 4,76 & 7,0 & 0,0211 & 95,24 \\
\hline $\begin{array}{l}\text { IL-4590CT/IL-6174GG/ } \\
\text { TNFA863CA }\end{array}$ & 15,00 & 1,61 & 9,7 & 0,0432 & 98,39 \\
\hline IL-4590CT/IL-6174GC/IL1B/31CC & 14,29 & 1,59 & 9,5 & 0,0465 & 98,41 \\
\hline $\begin{array}{l}\text { IL-6174GC/IL-10592CA/ } \\
\text { TNFA238GC }\end{array}$ & 33,33 & 12,70 & 4,2 & 0,0387 & 87,30 \\
\hline $\begin{array}{l}\text { IL-6174GG/IL-10592CA/ } \\
\text { TNFA863CA }\end{array}$ & 20,00 & 1,61 & 10,9 & 0,0116 & 98,39 \\
\hline $\begin{array}{l}\text { IL-6174GG/TNFA238GG/ } \\
\text { TNFA308GA }\end{array}$ & 14,29 & 1,59 & 9,5 & 0,0465 & 98,41 \\
\hline $\begin{array}{l}\text { IL-6174GG/TNFA238GG/ } \\
\text { TNFA863CA }\end{array}$ & 30,00 & 4,84 & 7,9 & 0,0055 & 95,16 \\
\hline $\begin{array}{l}\text { IL-6174GG/TNFA863CA/IL- } \\
\text { 1B31TT }\end{array}$ & 25,00 & 3,23 & 8,9 & 0,0084 & 96,77 \\
\hline $\begin{array}{l}\text { IL-4590CT/IL-6174GC/IL- } \\
\text { 10592CA/TNFA238GG }\end{array}$ & 23,81 & 4,76 & 7,0 & 0,0211 & 95,24 \\
\hline $\begin{array}{l}\text { IL-4590CT/IL-6174GC/IL- } \\
\text { 10592CA/TNFA308GA }\end{array}$ & 14,29 & 1,59 & 9,5 & 0,0465 & 98,41 \\
\hline $\begin{array}{l}\text { IL-4590CT/IL-6174GC/IL- } \\
\text { 10592CA/TNFA863CC }\end{array}$ & 20,00 & 1,61 & 10,9 & 0,0116 & 98,39 \\
\hline $\begin{array}{l}\text { IL-4590CT/IL-6174GG/ } \\
\text { IL10592CC/IL-1B31TC }\end{array}$ & 14,29 & 1,59 & 9,5 & 0,0465 & 98,41 \\
\hline
\end{tabular}


Таблица 4 (продолжение)

\begin{tabular}{|c|c|c|c|c|c|}
\hline \multirow{2}{*}{ Комбинации аллелей } & \multicolumn{2}{|c|}{$\begin{array}{c}\text { Частота среди больных РА } \\
\text { (в \%) } \\
\end{array}$} & \multirow{2}{*}{$\begin{array}{l}\text { Прогности- } \\
\text { ческий ко- } \\
\text { эфрфициент }\end{array}$} & \multirow{2}{*}{$\begin{array}{l}\text { Значимость } \\
\text { различий (р) }\end{array}$} & \multirow{2}{*}{$\begin{array}{l}\text { Специфич } \\
\text { ность }\end{array}$} \\
\hline & $\begin{array}{l}\text { нежелатель- } \\
\text { ный эффрект }\end{array}$ & отсутствие & & & \\
\hline $\begin{array}{l}\text { IL-4590CT/IL-6174GG/ } \\
\text { TNFA238GG/TNFA863CA }\end{array}$ & 15,00 & 1,61 & 9,7 & 0,0432 & 98,39 \\
\hline $\begin{array}{l}\text { IL-4590CT/IL-6174GC/ } \\
\text { TNFA238GG/IL-1B31CC }\end{array}$ & 14,29 & 1,59 & 9,5 & 0,0465 & 98,41 \\
\hline $\begin{array}{l}\text { IL-4590CT/IL-6174GG/ } \\
\text { TNFA308GG/TNFA863CA }\end{array}$ & 15,00 & 1,61 & 9,7 & 0,0432 & 98,39 \\
\hline $\begin{array}{l}\text { IL-4590CT/IL-6174GG/ } \\
\text { TNFA863CA/IL-1B31TC }\end{array}$ & 15,00 & 0,00 & 13,3 & 0,0129 & 100,0 \\
\hline $\begin{array}{l}\text { IL-6174GG/IL-10592CA/ } \\
\text { TNFA238GG/TNFA863CA }\end{array}$ & 20,00 & 1,61 & 10,9 & 0,0116 & 98,39 \\
\hline $\begin{array}{l}\text { IL-6174GG/IL-10592CA/ } \\
\text { TNFA863CA/IL-1B31TT }\end{array}$ & 20,00 & 1,61 & 10,9 & 0,0116 & 98,39 \\
\hline $\begin{array}{l}\text { IL-6174GG/TNFA238GG/ } \\
\text { TNFA863CA/IL-1B31TT }\end{array}$ & 25,00 & 1,61 & 11,9 & 0,0029 & 98,39 \\
\hline $\begin{array}{l}\text { IL-6174GG/TNFA308GG/ } \\
\text { TNFA863CA/IL-1B31TT }\end{array}$ & 15,00 & 1,61 & 9,7 & 0,0432 & 98,39 \\
\hline $\begin{array}{l}\text { IL-4590CT/IL-6174GC/ } \\
\text { IL-10592CA/TNFA238GA/ } \\
\text { TNFA308GA }\end{array}$ & 14,29 & 1,59 & 9,5 & 0,0465 & 98,41 \\
\hline $\begin{array}{l}\text { IL-4590CT/IL-6174GC/ } \\
\text { IL-10592CA/TNFA238GA/ } \\
\text { TNFA863CA }\end{array}$ & 20,00 & 1,61 & 10,9 & 0,0116 & 98,39 \\
\hline $\begin{array}{l}\text { IL-4590CT/IL-6174GC/ } \\
\text { IL-10592CA/TNFA238GA/ } \\
\text { IL-1B31TC }\end{array}$ & 14,29 & 1,59 & 9,5 & 0,0465 & 98,41 \\
\hline $\begin{array}{l}\text { IL-4590CT/IL-6174GC/ } \\
\text { IL-10592CA/TNFA308GA/ } \\
\text { TNFA863CA }\end{array}$ & 15,00 & 1,61 & 9,7 & 0,0432 & 98,39 \\
\hline $\begin{array}{l}\text { IL-4590CT/IL-6174GC/ } \\
\text { IL-10592CA/TNFA308GA/ } \\
\text { IL1B31TC }\end{array}$ & 14,29 & 1,59 & 9,5 & 0,0465 & 98,41 \\
\hline $\begin{array}{l}\text { IL-4590CT/IL-6174GC/ } \\
\text { TNFA238GA/TNFA308GA/ } \\
\text { TNFA863CA }\end{array}$ & 15,00 & 1,61 & 9,7 & 0,0432 & 98,39 \\
\hline $\begin{array}{l}\text { IL-4590CT/IL6174GC/ } \\
\text { TNFA238GA/TNFA863CA/IL- } \\
\text { 1B31TC }\end{array}$ & 15,00 & 0,00 & 13,3 & 0,0129 & 100,0 \\
\hline $\begin{array}{l}\text { IL-4590CT/IL6174GC/TNA308GA/ } \\
\text { TNFA863CA/IL-1B31TC }\end{array}$ & 15,00 & 0,00 & 13,3 & 0,0129 & 100,0 \\
\hline $\begin{array}{l}\text { IL-6174GC/IL10592CA/ } \\
\text { TNFA238GA/TNFA863CA/ } \\
\text { IL-1B31TC }\end{array}$ & 20,00 & 1,61 & 10,9 & 0,0116 & 98,39 \\
\hline $\begin{array}{l}\text { IL-6174GC/TNFA238GA/ } \\
\text { TNFA308GA/TNFA863CA/ } \\
\text { IL-1B31TC }\end{array}$ & 15,00 & 1,61 & 9,7 & 0,0432 & 98,39 \\
\hline
\end{tabular}


Таблица 4 (окончание)

\begin{tabular}{|c|c|c|c|c|c|}
\hline \multirow{2}{*}{ Комбинации аллелей } & \multicolumn{2}{|c|}{$\begin{array}{c}\text { Частота среди больных РА } \\
\text { (в \%) }\end{array}$} & \multirow{2}{*}{$\begin{array}{l}\text { Прогности- } \\
\text { ческий ко- } \\
\text { эфффициент }\end{array}$} & \multirow{2}{*}{$\begin{array}{l}\text { Значимость } \\
\text { различий (р) }\end{array}$} & \multirow{2}{*}{$\begin{array}{l}\text { Специфич- } \\
\text { ность }\end{array}$} \\
\hline & $\begin{array}{l}\text { нежелатель- } \\
\text { ный эффрект }\end{array}$ & отсутствие & & & \\
\hline $\begin{array}{l}\text { IL-4590CT/IL6174GC/IL10592CA/ } \\
\text { TNFA238GA/TNFA308GA/ } \\
\text { TNFA863CA }\end{array}$ & 15,00 & 1,61 & 9,7 & 0,0432 & 98,39 \\
\hline $\begin{array}{l}\text { IL-4590CT/IL-6174GC/ } \\
\text { IL-10592CA/TNFA238GA/ } \\
\text { TNFA308GA/IL-1B31TC }\end{array}$ & 14,29 & 1,59 & 9,5 & 0,0465 & 98,41 \\
\hline $\begin{array}{l}\text { IL-4590CT/IL6174GC/ } \\
\text { TNFA238GA/TNFA308GA/ } \\
\text { TNFA863CA/IL-1B31TC }\end{array}$ & 15,00 & 0,00 & 13,3 & 0,0129 & 100,0 \\
\hline
\end{tabular}

с тенденцией к повышению частоты аллелей генов IL-4 и IL-10, обеспечивающих высокий уровень продукции противовоспалительных цитокинов.

Напротив, в группе пациентов, не ответивших на базисную терапию, преобладают аллели генов, ассоциированные с высокой продукцией IL-1, низкой продукцией IL-6 и низкой продукцией цитокинов с антивоспалительной активностью.

При назначении любой терапии, особенно столь активной, как DMARDs, врач вправе ожидать появления побочных нежелательных эффектов. Мы проанализировали частоты встречаемости аллелей генов цитокинов в группе пациентов лишь с серьезными нежелательными явлениями на проводимую терапию, которые служат основанием для ее прекращения или серьезной коррекции (табл. 3). При анализе результатов в данных выборках пациентов нами также проанализированы частоты встречаемости 16362 возможных сочетаний генотипов 2-7 локусных комбинаций, из которых достоверно различающимися (p $<0,05)$ оказались 6 комбинаций.

У части пациентов РА, в геноме которых выявлены определенные комбинации аллелей генов цитокинов, часто выявляются серьезные нежелательные реакции при проведении DMARDs терапии, что должно заранее настраивать врача на использование минимально эффективных доз препаратов и на проведение профилактических мероприятий.

В генотипах данной группы пациентов, как правило, выявлены гетерозиготные варианты гаплотипов, включающие аллели, ассоциированные как с высоким, так и с низким уровнем продукции цитокинов с провоспалительной и антивоспалительной активностью. Возможно, именно эта «нестабильность» цитокинового генома является одним из факторов, способствующим развитию неадекватных реакций на противовоспалительную терапию.

Кроме того фиксируются нежелательные реакции минимальной выраженности при лечении иммуносупрессивными препаратами, которые носят кратковременный скоропроходящий характер. Для того, чтобы определиться с прогнозом возможного формирования серьезных или минимально выраженных нежелательных реакций на начало проведения базисной противоревматической терапии мы отдельно проанализировали генотипы в группе из 21 пациента, у которых были выявлены побочные реакции на лечение, не потребовавшие отмены лекарственного средства (табл. 4). При анализе результатов в данных выборках пациентов нами проанализированы частоты встречаемости 16362 возможных сочетаний генотипов 2-7 локусных комбинаций, из которых достоверно различающимися $(\mathrm{p}<0,05)$ при использовании одностороннего варианта точного метода Фишера оказалось 40, а при использовании двустороннего варианта - 36 комбинаций генотипов.

Обращает на себя внимание, что число достоверно ассоциированных комбинаций генотипов, ассоциированных с легкими нежелательными реакциями на лечение гораздо выше, чем число ассоциированных с серьезными нежелательными событиями, потребовавшими смены курса терапии. На наш взгляд эти данные могут быть использованы в качестве дополнительных лабораторных признаков, позволяюших прогнозировать формирование нежелательного явления различной степени выраженности. 


\section{Обсуждение}

Прогресс технологии генотипирования и разработка современных биоинформационных методов предоставили новые возможности для изучения роли вариабельности генома в развитии мультифакториальных заболеваний человека, ярким примером которых является PA. Разработанные на сегодняшний день каталоги генетических вариаций и структуры гаплотипов генома человека, обеспечивают основу для изучения действия естественного отбора в геноме человека и понимания различий в подверженности к РА. Данные, полученные в ходе реализации программы «НарМар», показали, что подверженность индивидов к мультифакториальным состояниям является частичным следствием эволюционного наследия человека. В связи с чем, изучение структуры неравновесия по сцеплению, отражающей совокупную историю действия демографических и эволюционных факторов, в различных популяциях играет немаловажную роль в понимании причин развития широко распространенных заболеваний, и способствует определению оптимальных стратегий обнаружения генетических вариантов подверженности к РА.

Полученные нами данные являются одним из возможных генетических факторов, участвующих в формировании ответа на проводимую базисную противоревматическую терапию. Цитокиновый геном индивида является конституционально стабильным базисом, формирующим баланс факторов регуляции воспалительного процесса. Показано, что эффекты отдельных полиморфизмов, обнаруживаемые при анализе ассоциаций, как правило, невелики и могут быть связаны не с самим изучаемым маркером, а со сцепленным с ним функционально значимым вариантом. Исходя из этого, нами использован метод комплексного анализа выявления в геноме пациента одновременно целого набора аллельных вариантов генов цитокинов, полиморфизм которых сосредоточен в регуляторных промоторных участках, содержащих сайты связывания транскрипционных факторов. Нами были проанализированы частоты встречаемости всех одно-, двух-, трех-, четырех-, пяти- , шести- и семилокусных комбинаций генотипов в группах с различными эффектами от проведенной терапии по результатам 24 недельного наблюдения.

Становится очевидным, что у пациента, в геноме которого преобладают аллельные вариан- ты генов провоспалительных цитокинов, ассоциированных с высоким уровнем их продукции лимфоидными и макрофагальными клетками, формируется один тип ответа на иммуносупрессивную и противовоспалительную терапию. Совсем другой тип реакции на болезньмодифицирующую терапию мы наблюдаем у пациентов с цитокиновым геномом, обеспечивающим преобладание аллельных вариантов генов, ассоциированных с высоким уровнем продукции антивоспалительных цитокинов.

В практической ревматологии вполне реально использовать полученные данные в качестве прогностических признаков, позволяющих до назначения DMARDs терапии предвидеть ее результаты и внести заранее необходимые коррективы, не используя напрасно ограниченное во времени «окно терапевтических возможностей». Для реализации таких возможностей необходимо обратиться к величинам прогностических коэффициентов, представленным в таблицах, в которых после сплошного анализа всех возможных комбинаций, представлены лишь достоверно различающиеся комбинации генотипов цитокинов. Достоверность прогноза предполагаемого эффекта от назначаемого лечения напрямую связана с величиной прогностического коэффициента. При достижении данной величины 12,8 вероятность реализации прогноза достигает 95\%, а при достижении порогового значения 19,8 до 99\%. Соответственно этой калибровочной кривой можно рассчитать вероятность правильного прогноза для любого полученного исследователем показателя прогностического коэффициента. Кроме того, математические преобразования в данном способе анализа позволяют суммировать логарифмированные значения величин прогностических коэффициентов и при использовании расширенных генетических комплексных показателей суммировать их значения для одного пациента и получать индивидуальные показатели с гораздо более высокими значениями. Необходимо также обратить внимание на высокие (доходящие до 100\%) показатели специфичности выявления ряда генетических комбинаций при сопоставлении анализируемых выборок. Столь редкие в ревматологии и в медицинской иммунологии в целом показатели специфичности позволяют, после проведения многоцентровых исследований, рассчитывать на их реальную клиническую значимость. 
Несомненно, полученные на сегодня данные, представленные в настоящей публикации, являются не окончательными. В настоящее время активно продолжается набор клинического материала, которых позволит не только существенно увеличить анализируемые выборки, но и сформировать новые группы пациентов с незначительным, но значимым клиническим эффектом проведенного лечения, с незначительно выраженными побочными реакциями на терапию и т.п. Формирующийся «банк данных» длительного хранения образцов ДНК пролеченных пациентов и ведение электронного регистра пациентов, позволят не только расширять группы обследованных лиц, но и существенно расширить перечень анализируемых полиморфизмов.

Проблема выбора наиболее высокоэффективной терапии именно для этого пациента имеет кроме чисто терапевтического, еще и фармакоэкономический и этический аспекты, в связи с высокой стоимостью применения нового поколения генно-инженерных биологических препаратов. С одной стороны, врач не имеет морального права отказать пациенту в назначении теоретически высокоэффективного препарата, но, с другой стороны, нет никакого смысла в назначении этих препаратов пациентам, в геноме которых содержатся комбинации аллелей генов цитокинов, заведомо обрекающие пациента на отсутствие эффекта от высокозатратной терапии в сочетании с потерей окна терапевтических возможностей от применения других схем терапии.

\section{Список литературы}

1. Вейр Б. Анализ генетических данных: Дискретные генетические признаки: пер. с англ. М.: Мир, 1995. - 400 с.

2. Власов В.В. Эффективность диагностических исследований. - М.: Медицина, 1988. $245 \mathrm{c}$.

3. Гланц С. Медико-биологическая статистика: пер. с англ. - М.: Практика, 1998. - 459 с.

4. Губарь Е.Е., Бочкова А.Г., Бунчук Н.В. Сравнительная эффективность и переносимость тройной базисной терапии (метотрексат + сульфасалазин + гидроксихлорохин) с монотерапией метотрексатом у больных ревматоидным артритом (предварительные результаты) // Тер. архив. - 2008. - № 5. - С. 25-30.
5. Гублер Е.В. Вычислительные методы анализа и распознавания патологических процессов. - Л.: Медицина, 1983. - 296 с.

6. Коненков В.И. Медицинская и экологическая иммуногенетика. - СО РАМН, Новосибирск, 1999. - 250 с.

7. Ластед Л.Б. Введение в проблему принятия решений в медицине. - М.: Мир, 1971. $282 \mathrm{c}$.

8. Насонов Е.Л. Иммунопатология ревматических заболеваний // Инновационные технологии в диагностике и лечении ревматических заболеваний: Лекции цикла повышения квалификации врачей. - Москва, 2009. - С. 4-10.

9. Реброва О.Ю. Статистический анализ медицинских данных. Применение пакета прикладных программ Statistica. - М.: Медиасфера, 2002. - 312 c.

10.Смольникова М.В., Соловьева И.Г., Коненкова Л.П., Сизиков А.Э., Герцог О.А., Кожевников В.С., Коненков В.И. Распределение аллельных вариантов генов цитокинов (TNFA и IL-10) у больных ревматоидным артритом европеоидов // Медицинская иммунология. 2007. - T. 9, № 2-3. - С. 204-205.

11.Фишер Р.А. Статистические методы для исследователей. - М.: Госстатиздат, 1958. $268 \mathrm{c}$.

12. Юнкеров В.И., Григорьев С.Г. Математикостатистическая обработка данных медицинских исследований. - СПб.: ВМедА, 2002. - 266 с.

13. Asghar T., Yoshida S., Kennedy S., Negoro K., Zhuo W., Hamana S., Motoyama S., Nakago S., Barlow D., Maruo T. The tumor necrosis factoralpha promoter $-1031 \mathrm{C}$ polymorphism is associated with decreased risk of endometriosis in a Japanese population // Human Reproduction. - 2004. Vol. 19, N 11. - P. 2509-2514.

14. Choi E., Lee H.J., Yoo T., Chanock S. A common haplotype of interleukin-4 gene IL-4 is associated with severe respiratory syncytial virus disease in Korean children // The Journal of Infectious Diseases. - 2002. - Vol. 186. - P. 1207 1211.

15.Coenen M.J., Toonen E.J., Scheffer H., RadstakeT.R., Barrera P., FrankeB. Pharmacogenetics of anti-TNF treatment in patients with rheumatoid arthritis // Pharmacogenomics. - 2007. - N 8. P. 761-773.

16. Combe B. Early rheumatoid arthritis: strategies for prevention and management // Best 
Pract. Res. Clin. Rheumatol. - 2007. - Vol. 21, N 1. P. 27-42.

17.Dieude P., Cornelis F. Genetic basis of rheumatoid arthritis // Jt. Bone Spine. - 2005. Vol. 9. - P. 3-7.

18. Fernandez-Real J.M., Vendrell J., Richart C., Gutierrez C., Ricart W. Platelet count and Interleukin 6 Gene polymorphism in healthy subjects // BMC Medical Genetics. - 2001. - Vol. 2, N 6. - P. 14712350 .

19. Fleischman R.M. Is there a need for new therapies for rheumatoid arthritis // J. Rheumatol. 2005. - Vol 73, N 32. - P. 3-7.

20.Fransen J., Creemers M.C., Van Riel P.L. Remission in rheumatoid arthritis: agreement of the disease activity score (DAS28) with the ARA preliminary remission criteria // Rheumatology (Oxford). - 2004. - N 43. - P. 1252-1255.

21. Goldblatt F., Isenberg D.A. New therapy for rheumatoid arthritis // Clin. Exp. Immunol. 2005. - N 140. - P. 195-204.

22.Haukim N., Bidwell J.L., Smith A.J.P., Keen L.J., Gallagher G., Kimberly R., Huizinga T., McDermott M.F., Oksenberg J., McNicholl J., Pociot F., Hardt C., D'Alfonso S. Cytokine gene polymorphism in human disease:on-line databases // Genes and Immunity. - 2002. - Vol. 3, N 6. P. 313-330.

23.John S., Myerscough A., Marlow A., Hajeer A., Silman A., Ollier W., Worthington J. Linkage of cytokine genes to rheumatoid arthritis. Evidence of genetic heterogeneity // Ann. Rheum. Dis. 1998. - Vol. 57, N 6. - P. 361-365. Li H.Q., Li Z., Liu Y., Li J.H., Dong J.Q., Gao J.R., Gou C.Y., Li H. Association of polymorphism of tumor necrosis factor-alpha gene promoter region with outcome of hepatitis B virus infection // World J. Gastroenterol. - 2005. - Vol. 11, N 33. - P. 52135217.

24. Mehta C.R., Patel N.R., Gray R. On computing an exact confidence interval for the common odds ratio in several $2 \times 2$ contingency tables // JASA. - 1985. - Vol. 80. - P. 969-973.

25. Mock C.C., Lanchbury J.S., Chan D.W., Lau C.S. Interleukin-10 promoter polymorphisms in Southern Chinese patients with systemic lupus erythematosus // Arthritis Rheum. - 1998. Vol. 41. - P. 1090-1095.

26.O'Dell J.R., Leff R., Paulsen G., Yaire C. Treatment of rheumatoid arthritis with methotrexate and hydroxychloroquine, methotrexate and sulfasalazine, or a combination of three medications // Arthr. And Rheum. - 2002. Vol. 46. - P. 1164-1170.

27.O’Rielly D.D., Roslin N.M., Beyene J., Pope A., Rahman P. TNF-alpha-308 G/A polymorphism and responsiveness to TNF-alpha blockade therapy in moderate to severe rheumatoid arthritis: a systematic review and meta-analysis // Pharmacogenomics J. - 2009. - N 9. - P. 161-167.

28. Pascual M., Nieto A., Mataraán L., Balsa A., Pascual-Salcedo D., Martín J. IL-6 promoter polymorphisms in rheumatoid arthritis // Genes and Immunity. - 2000.- N 1. - P. 338-340.

29. Patino-Garcia A., Sotillo-Pineiro E., Modesto C., Sierrasesumaga L. Screening of the human tumor necrosis factor-alpha (TNF-alpha) gene promoter polymorphisms by PCR-DGGE analysis // Mutation Research. - 1999. - Vol. 406. P. 121-125.

30.Pincus T., Yazici Y., Sokka T., Alehata D., Smolen J.S. Methotrexate as the "anchor drug" for the treatment of early rheumatoid arthritis // Clin. Exp. Rheumatol. - 2003. - Vol. 21, N 31. P. 179-185.

31.Quinn M.A., Conaghan P.G., Emery P. The therapeutic approach of early intervention for rheumatoid arthritis: what is the evidence? // Rheumatology (Oxford). - 2001. - N 40. - P. 12111220.

32. Ranganathan P. An Update on Methotrexate Pharmacogenetics in Rheumatoid Arthritis // Pharmacogenomics. - 2008. - Vol. 4, N 9. - P. 439451 .

33.Seitz M., Wirthmüller U., Möller B., Villiger P.M. The -308 tumour necrosis factor-alpha gene polymorphism predicts therapeutic response to TNFalpha-blockers in rheumatoid arthritis and spondyloarthritis patients // Rheumatology (Oxford). - 2007. - N 46. - P. 93-96.

34.Steinbrocker O., Traeger C.N. Therapeutic criteria in rheumatoid arthritis // J. Am. Med. Association. - 1949. - Vol 8, N 40. - P. 659-662.

35. Warlé M.C., Farhan A., Metselaar H.J., Hop W.C., Perrey C., Zondervan P.E., Kap M., Kwekkeboom J., Ijzermans J.N., Tilanus H.W., Pravica V., Hutchinson I.V., Bouma G.J. Are Cytokine Gene Polymorphisms Related to in vitro cytokine production profiles? // Liver Transplantation. - 2003. - Vol. 9, N 2. - P. 170-181.

36. Wessels J. A. M., de Vries-Bouwstra J.K., Heijmans B.T., Slagboom P.E., Methotrexate 
in Early Rheumatoid Arthritis Are Associated With Single-Nucleotide Polymorphisms in Genes Coding for Folate Pathway Enzymes // Arthritis \& Rheumatism. - 2006. - Vol. 54, N 4. - P. 1087-1095. 37.Zhang D., Zheng H., Zhou Y., Tang X., Yu B., Li J. Association of IL-1beta gene polymorphism with cachexia from locally advanced gastric cancer // BMC Cancer. - 2007. - Vol. 7, N 45. - P. 45-51.

поступила в редакцию 08.02.2010

отправлена на доработку 12.02.2010

принята к печати 27.02.2010 